\title{
Köpfer, Andreas
}

\section{'Distant Bodies - Collective Spaces - Borders'. Herstellung und} Aneignung von Lernräumen in Zeiten erforderlicher Distanzierung

Krause, Sabine [Hrsg.]; Breinbauer, Ines Maria [Hrsg.]; Proyer, Michelle [Hrsg.]: Corona bewegt - auch die Bildungswissenschaft. Bildungswissenschaftliche Reflexionen aus Anlass einer Pandemie. Bad Heilbrunn : Verlag Julius Klinkhardt 2021, S. 103-115

\section{Quellenangabe/ Reference:}

Köpfer, Andreas: 'Distant Bodies - Collective Spaces - Borders'. Herstellung und Aneignung von Lernräumen in Zeiten erforderlicher Distanzierung - In: Krause, Sabine [Hrsg.]; Breinbauer, Ines Maria [Hrsg.]; Proyer, Michelle [Hrsg.]: Corona bewegt - auch die Bildungswissenschaft.

Bildungswissenschaftliche Reflexionen aus Anlass einer Pandemie. Bad Heilbrunn : Verlag Julius Klinkhardt 2021, S. 103-115 - URN: urn:nbn:de:0111-pedocs-222270 - DOI: 10.25656/01:22227

https://nbn-resolving.org/urn:nbn:de:0111-pedocs-222270

https://doi.org/10.25656/01:22227

in Kooperation mit / in cooperation with:

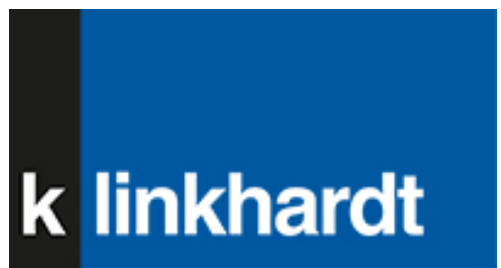

http://www.klinkhardt.de

\section{Nutzungsbedingungen}

Dieses Dokument steht unter folgender Creative Commons-Lizenz: http://creativecommons.org/licenses/by-nc-sa/4.0/deed.de - Sie dürfen das Werk bzw. den Inhalt unter folgenden Bedingungen vervielfältigen, verbreiten und öffentlich zugänglich machen sowie Abwandlungen und Bearbeitungen des Werkes bzw. Inhaltes anfertigen: Sie müssen den Namen des Autors/Rechteinhabers in der von ihm festgelegten Weise nennen. Dieses Werk bzw. der Inhalt darf nicht für kommerzielle Zwecke verwendet werden. Die neu entstandenen Werke bzw. Inhalte dürfen nur unter Verwendung von Lizenzbedingungen weitergegeben werden, die mit denen dieses Lizenzvertrages identisch oder vergleichbar sind.

Mit der Verwendung dieses Dokuments erkennen Sie die Nutzungsbedingungen an.

\section{Terms of use}

This document is published under following Creative Commons-License: http://creativecommons.org/licenses/by-nc-sa/4.0/deed.en - You may copy, distribute and transmit, adapt or exhibit the work in the public and alter, transform or change this work as long as you attribute the work in the manner specified by the author or licensor. You are not allowed to make commercial use of the work. If you alter, transform, or change this work in any way, you may distribute the resulting work only under this or a comparable license.

By using this particular document, you accept the above-stated conditions of use.

\section{Kontakt / Contact:}

\section{peDOCs}

DIPF | Leibniz-Institut für Bildungsforschung und Bildungsinformation Informationszentrum (IZ) Bildung

E-Mail: pedocs@dipf.de

Internet: www.pedocs.de

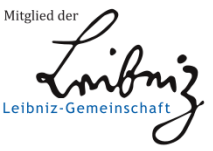




\section{Sabine Krause}

Ines Maria Breinbauer

Michelle Proyer

(Hrsg.)

\section{Corona bewegt - auch die Bildungswissenschaft}

Bildungswissenschaftliche Reflexionen aus Anlass einer Pandemie 
Krause / Breinbauer / Proyer Corona bewegt - auch die Bildungswissenschaft 


\section{Sabine Krause \\ Ines Maria Breinbauer \\ Michelle Proyer \\ (Hrsg.)}

\section{Corona bewegt - auch die Bildungswissenschaft}

Bildungswissenschaftliche Reflexionen aus Anlass einer Pandemie 
Die Veröffentlichung der Open Access-Publikation wurde durch Mittel der Universität Innsbruck und der Universität Wien möglich.

Dieser Titel wurde in das Programm des Verlages mittels eines Peer-Review-Verfahrens aufgenommen. Für weitere Informationen siehe www.klinkhardt.de.

Bibliografische Information der Deutschen Nationalbibliothek Die Deutsche Nationalbibliothek verzeichnet diese Publikation in der Deutschen Nationalbibliografie; detaillierte bibliografische Daten sind im Internet abrufbar über http://dnb.d-nb.de.

2021.h. () by Julius Klinkhardt.

Foto Umschlagseite 1: () Sabine Krause, Innsbruck.

Druck und Bindung: AZ Druck und Datentechnik, Kempten.

Printed in Germany 2021.

Gedruckt auf chlorfrei gebleichtem alterungsbeständigem Papier.

(c) (i)(5) Die Publikation (mit Ausnahme aller Fotos, Grafiken und Abbildungen) ist veröffent-

licht unter der Creative Commons-Lizenz: CC BY-NC-SA 4.0 International

https://creativecommons.org/licenses/by-nc-sa/4.0/

ISBN 978-3-7815-5889-2 digital

doi.org/10.35468/5889

ISBN 978-3-7815-2453-8 


\section{Inhalt}

Ines M. Breinbauer, Sabine Krause und Michelle Proyer

Corona bewegt - nicht nur, aber auch die Bildungswissenschaft

Barbara Platzer

Verantwortung in der Pandemie. Über die Unzulänglichkeit individueller

Verantwortung und blinde Flecken des Pandemie-Diskurses

Thorsten Fuchs und Dominik Matzinger

Lost in Pandemic. Zur Frage von adoleszenten Bildungsprozessen und

Übergängen in Zeiten des Social Distancing

Ines M. Breinbauer

Lernen an den Grenzen unseres Wissens

\section{Dominic Lüthi}

,Corona' im Ethikunterricht

Sabine Krause

Ver(un)sichern in Zeiten der Ver(un)sicherung

Andreas Köpfer

,Distant Bodies - Collective Spaces - Borders': Herstellung und

Aneignung von Lernräumen in Zeiten erforderlicher Distanzierung

Johannes Reitinger und Michelle Proyer

Studentisches Lernen im digitalen Raum in Zeiten der Pandemie

Tina Obermayr, Seyda Subasi Singh, Gertraud Kremsner,

Oliver Koenig und Michelle Proyer

Revisiting vulnerabilities - Auswirkungen der Pandemie auf die

$(\mathrm{Re})$ Konstruktion von Vulnerabilität*en im Kontext von Bildung

Franziska Felder

Corona und die ultimativen ,memento mori‘. Überlegungen zu einem besseren Umgang mit behinderungsbedingten Vulnerabilitäten in Demokratie und Bildung

Autor*innenspiegel 
Andreas Köpfer

\section{,Distant Bodies - Collective Spaces - Borders': Herstellung und Aneignung von Lernräumen in Zeiten erforderlicher Distanzierung}

\section{Einleitung - Zur Verfasstheit von Bildung in Zeiten erforderlicher Distanzierung}

Durch die gesamtgesellschaftliche Reaktion auf die Corona-Pandemie wurde neben einer wirtschaftlichen Krise auch ein abrupter, gemeinhin als krisenhaft empfundener Stillstand des Präsenzbetriebs innerhalb von Bildungsorganisationen hervorgerufen. Unter politischer Vorgabe physischer Distanzierung wurden in vielen Ländern zunächst die Prozesse formaler Bildung in die Familien bzw. häusliche Situation hinein verlagert (z.B. durch sog. Home-Schooling ${ }^{1}$ ), bevor sukzessive Wiederöffnungsmaßnahmen von Bildungsorganisationen angestrebt wurden - abgelöst von Phasen wechselnder Lockdowns, die zu einer notgedrungenen organisatorischen Umgestaltung bislang routinehafter Praxis führten und führen.

Ohne an dieser Stelle die Krisensituation, die für viele Menschen tödliche Folgen hat, für wissenschaftliche Explorationszwecke zu instrumentalisieren, soll ein notwendiger kritischer Blick auf die gesellschaftliche Schock-Reaktion im Sinne eines affektartigen Umgangs mit Bildung in Krisenzeiten gerichtet werden, um - und dies wird als Auftrag von Wissenschaft gesehen - empirische Perspektivierungen der veränderten und potenziell herausfordernden Lernsituation von Kindern und Jugendlichen zu leisten. Dies mit dem spezifischen Fokus auf potenzielle Lernbenachteiligungen und Marginalisierungen, die durch eine außerplanmäßige Unterbrechung des formalen Bildungsbetriebs in Schulen hervorgerufen werden könnten.

Das besondere Augenmerk dieses Beitrags liegt auf Lernräumen und Körpern, jedoch wird nicht die naheliegende Frage gestellt, was sich nun abrupt - z.B. durch Home-Schooling - verändert habe, sondern: Welche, in die Bildungsor-

1 Home-Schooling wird hier verstanden als Lehrkonzept zur Verlagerung des Unterrichts von der Schule in das Zuhause der Schüler*innen unter Verwendung distanzkonformer, u.a. digitaler Kommunikationswege. 
ganisation Schule eingeschriebenen, Konstellationen und Positionen der Körper hinsichtlich formaler Bildung fördert die aktuelle Raumkonstellation zutage? Wie werden hierin eingelagerte Barrieren und Benachteiligungen sichtbar?

Vor dem Hintergrund inklusionstheoretischer Überlegungen (Weisser 2017) sowie mit Bezug zu raum- wie körpersoziologischen Diskursen (Schroer 2003, 2012) werden die durch die Corona-Pandemie veränderten Raumkonstellationen skizziert. Weiter werden die Grundzüge eines derzeit laufenden explorativen Forschungsprojekts „Distant Bodies - Collective Spaces - Borders" ${ }^{2}$ vorgestellt und theoretisch wie methodologisch mit Blick auf die Visualisierung von Lernbedingungen und -räumen diskutiert.

\section{Ein (un)möglicher Kontextualisierungsversuch - die Corona-Pandemie und Deterritorialisierung in Bildungsorganisationen}

Wird von der aktuellen „Corona-Situation“ gesprochen, so wird ein einschneidendes, die aktuelle Praxis in Bildungsorganisationen unterbindendes Phänomen artikuliert - eine zeitlich unbestimmbare Phase der Erforderlichkeit physischer Distanz zur Wahrung von Hygiene- und Infektionsschutz. Während diese Ausgangslage sämtliche Lebens- und Handlungsbereiche betrifft, wird im Folgenden der Blick auf die Bildungsorganisation Schule gerichtet, die sich als organisatorische Einheit in spezifischen Rollen- und Raumkonstellationen (u.a. kollektive Präsenz, Aufmerksamkeitsverhältnis, unterschiedliche Lernsettings, längere gemeinsame Aufenthalte in spezifischen Räumen) ausdrückt und in vereinbarten und zueinander erfüllten Praktiken der Erziehung und Bildung prozessiert wird. Auf den ersten Blick erscheint die Corona-Pandemie, die sich in bildungspolitisch variierenden Regelungen zur Distanznahme ausdrückt, einer Unterbindung und Stilllegung schulisch-unterrichtlichen Praktiken gleichzukommen - insbesondere bezogen auf das gemeinsame Lernen in direkter sozialer Interaktion.

Gleichwohl kann die ,ruckartige' Suspendierung einer routinierten Handlungspraxis auch so gelesen werden, dass als Reaktion einige spezifische Bedürfnisse artikuliert wurden (z.B. die Qualifikationsfunktion von Schule und die ihrer Wahrung mittels summativer Bewertungsformen in einer möglichst regelkonformen Durchführung von Abschlussprüfungen) und einige nicht (z.B. die Sicherung

2 Das explorative Forschungsprojekt „Distant Bodies - Collective Spaces - Borders “ wurde gemeinsam mit Sabine Krause (Innsbruck), Michelle Proyer (Wien) und Raphael Zahnd (Basel) entwickelt. Die in diesem Artikel dargestellten Überlegungen zum Forschungsdesign und zu den methodologischen Perspektiven wurden hierin gemeinsam erarbeitet. Weitere Informationen zum Projekt finden sich auf der Projekthomepage www.distantbodies.eu [Stand: 24.02.2021]. 
des gemeinsamen Lernens und des Austauschs von Peers). Die Reaktion kann dahingehend dechiffriert werden, dass während der Corona-Pandemie manche raumbezogenen bildungswissenschaftlichen Diskurse (wie z.B. des Gemeinsamen Lernens, Bewegten Lernens, handlungsorientierten Unterrichts) unterbunden werden und andere (wie z.B. Diskurse um räumliche Differenzierung, selbstreguliertes Lernen, Deterritorialisierung, informelles Lernen, Digitalisierung etc.) ein höheres Maß an Anschlussfähigkeit aufzeigen.

Markant zeigt sich eine Art „Deterritorialisierung“ (Allan 2004) von formaler Bildung. Vormals kollektive Präsenz in Klassenräumen wird zugunsten einer räumlich flexibilisierten Lernsituation (z.B. im Home-Schooling) aufgelöst. Diese ,tektonische' Verschiebung von formaler Bildung in den Zwischenraum von Schule und Familie (vgl. Sliwka \& Klopsch 2020) wird begleitet durch ein hohes Maß an Neuaushandlung von Rollen und Zuständigkeiten, von Erreichbarkeiten und Abgrenzungen. Somit wird deutlich, dass durch die Corona-Pandemie nicht per se eine ,Entgrenzung' (Ahrens 2009) von Bildung prozessiert wird, sondern vielmehr, vor dem Hintergrund veränderter organisationaler Rahmenbedingungen, eine ,Verräumlichung, d.h. eine organisationale Herstellung von Raum (Soja 1985, 2008) mit den zur Verfügung stehenden (digitalen) Kommunikationsmitteln. In den Schutz- und Hygienemaßnahmen materialisieren sich dabei gesellschaftliche und pädagogische Vorstellungen von Bildung unter den Vorzeichen physischer Distanz und deren (körperlicher) Einhaltung durch die Bildungssubjekte. In den Bedingungen sind Vorstellungen von Raum und Körper im Sinne von Erwartungen verankert. Dies schließt an ein von Hummrich ausgedrücktes und auf Bourdieu (2016 [1985]) rekurrierendes Raumverständnis an, dass „materiale Raumgegebenheiten als in der Architektur verobjektivierte Ausdrucksgestalten sozialen Handelns zu sehen [sind, d.V.], die jedoch durch die Zeitlichkeit gebrochen und unter den Anforderungen des Wandels gebeugt [werden, d.V.]" (Hummrich 2009, 4). So kann schlussgefolgert werden, dass die Lernbedingungen der Kinder und Jugendlichen durch die Corona-Pandemie eine zeitlich beschleunigte Transformation erfahren, die - so ist erwartbar - die ohnehin raumzeitliche Verzerrung von sozialem und physischem Raum, ergo die Diskrepanz der Vorstellung von Bildung und deren Materialisierung, erweitern. Zusammengefasst führt die durch die Corona-Pandemie hergestellte Raumproduktion und deren Aneignung durch Kinder und Jugendliche somit zu einer zunächst erwartungswidrigen Lernsituation, die es empirisch zu perspektivieren gilt.

Die bislang wenig ausführliche Literatur- und Forschungslage im erziehungswissenschaftlichen Fachdiskurs zum (schulischen) Lernen in Zeiten erforderlicher Distanz, zu der der vorliegende Band beiträgt, widmet sich unterschiedlichen Feldern dieser veränderten Bildungssituation. Unter anderem werden Themen wie Digitalisierung, Technisierung und die Überlagerung von formaler und informeller Bildung im Home-Schooling in den Blick genommen. Zudem wird die Auf- 
merksamkeit auf die Auswirkungen der prekären Lernsituation auf bereits marginalisierte und von Aussonderung bedrohte Schüler*innengruppen gelenkt, u.a. Schüler*innen mit sonderpädagogischem Unterstützungsbedarf im Bereich Lernen (vgl. Goldan et al. 2020), Schüler*innen mit zugewiesener sprachlicher Förderung im Bereich DaZ/DaF (Gogolin 2020) oder Schüler*innen aus sozial(räumlich) und sozio-ökonomisch deprivierten und benachteiligten Lagen (Bremm \& Rachenbäumer 2020). Hier deuten sich - insbesondere bei vulnerablen Gruppen - starke psychische Belastungen an (vgl. Obermayr, Subasi Singh, Kremsner, Koenig \& Proyer sowie Felder in diesem Band). Dies verstärkt die Annahme, dass die erforderlichen Schutzmaßnahmen und die damit zusammenhängende Suspendierung des präsenten Lehrbetriebs an Schulen zu einer Reproduktion und Verstärkung sozialer Ungleichheit führe. Erste empirische Perspektiven unterstützen dies (vgl. Andresen et al. 2020). Gleichwohl besteht eine latente Gefahr der empirischen Reifizierung sozialer Asymmetrie durch die askriptive Positionierung von Personengruppen als benachteiligt. Daher ist es notwendig, den Blick auf die Handlungspraxis und auf den (ressourcenorientierten, ggf.) widerständigen und in jedem Falle handlungsmächtigen Umgang von Schüler*innen mit den ihnen zur Verfügung stehenden und von ihnen mit hergestellten Lernbedingungen zu richten (vgl. Kap. 4).

\section{Raum, Materialität und Handlung - die Sichtbarmachung von Inklusionsbedingungen in Lernräumen und -körpern}

Die Corona-Pandemie und räumliche Bezugnahmen zur Verfasstheit der Organisation von Bildung sind eng miteinander verbunden. Die zunächst naheliegende Referenz auf räumliche Distanzierung als Handlungsanforderung wird schnell um weitere physische wie sozial-räumliche Dimensionen erweitert, wenn Fragen der Unterbrechung peerbezogenen Lernens, potenzieller sozial-räumlicher Deprivation oder der Umgestaltung von unterrichtsbezogener Kommunikation und deren Verlagerung in digitale Räume aufgeworfen werden. So zeichnet sich ein Zusammenspiel neuer physischer und sozialer Raumkonstellationen ab, welche einer bildungswissenschaftlichen Reflexionsfläche bedürfen.

Ohne an dieser Stelle eine Einführung in die Pluralität von Referenzierungen von Raum in der erziehungswissenschaftlichen Fachdebatte geben zu können (vgl. u.a. Wulf \& Zirfas 2014), kann konstatiert werden, dass sich zunehmend sog. erziehungswissenschaftliche Space Studies (Glaser, Koller, Thole \& Krumme 2018) herausbilden, die im Anschluss an den ,spatial turn' (Döring \& Thielmann 2008) eine relationale Verschränkung von physischem und sozialem Raum vorsehen. Raum wird dabei weder als Container gedacht, in dem soziale Praxis stattfin- 
det, noch als sozialer Raum, dessen Praxis frei von physischer Beeinflussung stattfindet. Vielmehr wird Raum-Praxis als habitualisierte soziale Praxis verstanden, die durch physisch-materielle Grundgegebenheiten präformiert ist und zugleich durch Handlungen im sozialen Raum den physischen Raum mit hervorbringt. In ihrer ,Raumsoziologie‘ entwirft Löw (2001) Raum konsequent als soziales Produkt. Raum ist hier definiert als „eine relationale (An)Ordnung von Lebewesen und sozialen Gütern an Orten" $(2001,271)$. Es ist somit weniger die physischmaterielle Dinglichkeit, die Verhalten (mit-)erklären kann. Eher sind es die in materiellen Bedingungen enthaltenen Bedeutungen, welche „ein bestimmtes Verhalten nahelegen und ein anderes unterdrücken" (Schroer 2012, 177). Schroer konstatiert hier treffend: „Räume [...] strukturieren vor, in welche Situationen wir kommen können, welche Erwartungen wir haben können, sie strukturieren Interaktionsabläufe, machen einige wahrscheinlicher, andere unwahrscheinlich." (Ebd., 176)

Diese grundlegend an der räumlichen Konstitution und deren präfigurierender Wirkung ansetzende Raumperspektive wird in Schatzkis Theorie der Praxis weiter ausgeführt. Unter besonderer Fokussierung auf,prefiguration' (Schatzki 2019, 43) wird davon ausgegangen, dass Räume Handlungen vorstrukturieren und bedingen, ergo einen Einfluss auf das Raumhandeln von Akteur*innen haben, diese Handlungen jedoch nicht (strukturell) determinieren. Hier stehen Schatzkis raumtheoretische Annahmen in einer Linie mit denen Bourdieus (2018 [1991]), dessen Sozialraum-Konzept zwar eine Bedingtheit des Handelns durch die sozialräumliche Lage, gleichzeitig jedoch auch einen eigenaktiven Prozess der Aneignung zur Wahl stehender Räume und somit eine habituellen Herstellung von sozialem - und hieran angeschlossen auch physischem - Raum (vgl. Hummrich 2012; Köpfer, Lemmer \& Rißler 2021) vorsieht. Dies bezieht sich neben dem sozialen ebenso auf den körperlichen Vollzug (Alkemeyer 2006). Körperlichkeit wird somit nicht als naturalistisches Merkmal, sondern als soziales Phänomen erachtet (vgl. Bruner 2005), das in gesellschaftlichen Zusammenhängen „unweigerlich vergeschlechtlicht, sozial klassifiziert, ethnisch und kulturell codiert sowie Normalitäts- und Ästhetikdiskursen unterworfen wird" (Bruner 2005, 33). So werden Körper unter den Vorzeichen schulischer Bedingungen und darin enthaltener Differenzsetzungen (überwiegend ableistisch) entworfen. Diese Behandlungen schreiben sich in den Körper sowie in die Körperdiskurse ein und treten in spezifischen empirischen Dokumenten im Rahmen der Corona-Pandemie zutage, z.B. in Hygienekonzepten, Home-Schooling Maßnahmen etc. Am Beispiel von Home-Schooling, in dem eine Verschiebung der Ansprüche formaler Bildung in andere Sozialisationskontexte mit anderen Verhaltensanforderungen stattfindet, können so, neben materiellen Artefakten wie Möbeln, Schreibutensilien, technischen Geräten, auch Inkorporierungen der lernräumlichen Situation in den Blick genommen werden. Schüler*innen werden sozusagen für formale Bildung vor 
dem Hintergrund der aktuellen Distanzwahrung körperlich neu erschlossen und gleichzeitig vermessen Schüler*innen ihre Lernräume unter veränderten Bedingungen (Nentwig-Gesemann 2007). Sie richten ihren Körper aus, verorten ihn - bezogen auf die (kulturell) bedingte und unter der aktuellen Corona-Pandemie stehende Beeinflussung - und verhalten sich körperlich zu dieser neuen Lernsituation (Schroer 2003).

Dies wirft grundlegende Fragen nach der Organisation von Bildung und der ReAktualisierung von Grenzziehungen auf. Welche (räumlichen) Grenzen werden eingeführt, bestärkt, stillgestellt, abgebaut? Welche werden neu verhandelt? Welches sind bestehende, neu auferlegte oder stillschweigend weiterhin performierte Inklusionsbedingungen? Hier wird an ein sozialwissenschaftliches Verständnis von Inklusion und Exklusion angeknüpft, das in Nähe zu den sog. Disability Studies (vgl. Hirschberg \& Koebsell, 2021) und einer anti-essentialistischen Perspektive auf Behinderung steht (vgl. Weisser 2005, 2017). Es bezieht sich auf die Art und Weise, wie Interaktionszusammenhänge hergestellt werden und wie Akteur*innen innerhalb dieser relevant bzw. nicht-relevant gemacht werden. Entscheidend hierfür sind Inklusionsbedingungen im Sinne von Prämissen der Entscheidung, die organisational verankert sind (z.B. Zuweisung von sonderpädagogischem Unterstützungsbedarf) und stillschweigende Erwartungen an die Akteur*innen richten. Werden die Inklusionsbedingungen nicht erfüllt, sind Exklusionseffekte zu erwarten, wobei jedoch der „Status dieser Bedingungen [...] unklar“ ist (Weisser 2017, 145; Köpfer, Papke \& Gerdes 2019). Die Inklusionsbedingungen haben also impliziten Charakter und können daher nur in einer Beobachtung zweiter Ordnung sichtbar gemacht werden (ebd., 146f.).

\section{Empirische Perspektivierung - Beispielprojekt „Distant Bodies - Collective Spaces - Borders“}

Die vorangegangenen theoretischen Überlegungen machen deutlich, dass die empirische Erfassung der Lernsituation von Kindern und Jugendlichen unter veränderten räumlichen Bedingungen als Beobachtung zweiter Ebene zu erfolgen hat, um zugrundeliegende "Inklusionsbedingungen“ sichtbar zu machen, die potenziell mit der ,ruckartigen "Verräumlichung der Transformationsnotwendigkeit einhergingen und sich innerhalb der Lernbedingungen wiederfinden. Daher wird im Folgenden eine empirische Perspektivierung von Lernkörpern in Zeiten erforderlicher Distanz vorgestellt, die im Rahmen des Projekts „Distant Bodies Collective Spaces - Borders" (Köpfer, Krause, Proyer, Zahnd) aktuell bearbeitet wird und sich in der Forschungsdurchführung befindet. Wenngleich an dieser Stelle (noch) keine empirischen Ergebnisse präsentiert werden, soll die Skizzie- 
rung des Forschungsdesigns als Untermauerung der theoretischen Ausführungen der vorherigen Kapitel dienen. Das genannte Forschungsprojekt ist ein von an erziehungswissenschaftlichen und sonderpädagogischen Instituten angesiedelten (Inklusions-)Forscher*innen aus Österreich, der Schweiz und Deutschland durchgeführtes exploratives Projekt, das vergleichend die in Lernsituationen präsentierten Körper von Jugendlichen in unterschiedlichen Ländern und Kulturkreisen analysiert.

Ausgangspunkt und zugleich Erkenntnisinteresse ist dabei, wie die aktuelle Corona-Pandemie zu veränderten Lernräumen geführt hat und wie und in welchen Räumen Schüler*innen in Bildungsorganisationen vor dem Hintergrund der aktuellen Corona-Pandemie lernen bzw. wie sie ihre aktuelle lernräumliche Situation präsentieren und beschreiben. Der Annahme folgend, dass die durch die Corona-Schutzmaßnahmen erfolgten und nach wie vor erfolgenden (Teil-) Schließungen von Schulen zu einer abrupt veränderten Handlungspraxis der Schüler*innen hinsichtlich der Gestaltung und Ausübung von Lernen und der Einrichtung von Lernräumen geführt hat (sei dies innerhalb von Bildungseinrichtungen oder zuhause), werden in diesem Projekt also diese veränderten und/oder neu geschaffenen Lernräume in den Blick genommen. In diesen ,unüblichen' und vor dem Hintergrund von verordneten Schutzmaßnahmen hergestellten Räumen - so die weitere Annahme - bilden sich potenziell Spuren von Unsicherheit, Isolation, Prekarität und sozialer Ungleichheit sowie organisational vermittelte Inklusionsbedingungen $\mathrm{ab}$, die bereits in den Bildungs- und Erziehungsverhältnissen angelegt, jedoch durch die aktuellen abrupten Transformationsprozesse weniger verschleierbar sind und, visuell gesprochen, in dieser krisenhaften Zeit, durch die Schneedecke hervorbrechen' (Stumberger 2010).

Die Perspektivierung und Präsentation der Lernräume erfolgt visuell (mittels Fotografien und Fotogrammen) sowie textbasiert (durch verschriftlichte Beschreibungen der Kinder und Jugendlichen zu den Fotografien und Fotogrammen). Dabei steht die Perspektive der Kinder und Jugendlichen im Vordergrund, da sie als Bildproduzent*innen und zugleich Beschreibende ihrer Lernräume maßgeblich an der Produktion der Daten - und folglich an der Herstellung der visuellen Perspektivität - beteiligt sind. So werden, angelehnt an Goffman (2001), keine authentischen Tätigkeiten der Lernenden in den Lernräumen visuell dargestellt und beschrieben, sondern Re-Präsentationen von typischen Tätigkeiten, in denen - mit einer gewissen Zurschaustellung und Hybris - eine Reflexion der auch für die Lernenden abrupt erfolgten Veränderung ihrer Lernräume ermöglicht und dargestellt wird. Unter Anwendung von Methoden der visuellen Soziologie, erziehungswissenschaftlichen Bildforschung und qualitativ-rekonstruktiven Sozialforschung (u.a. auch spielerischer Methoden der partizipativen Forschung wie z.B. der Mannequin-Challenge) werden so visuelle und textbasierte Eindrücke dazu 
gesammelt und analysiert, wie Kinder und Jugendliche vor dem Hintergrund der aktuellen Pandemie-Situation sich in ihren Lernräumen darstellen (wollen).

Da die Corona-Pandemie eine globale Dimension besitzt und nicht an Länderoder Nationengrenzen Halt macht, ist es nahe liegend, die je nach sozialer, kultureller, politischer Ausgangslage eines jeden Landes/Kulturkreises unterschiedlichen Reaktionen auf die Corona-Pandemie im Bereich Bildung vergleichend zueinander ins Verhältnis zu setzen. Wie sich die Corona-Pandemie sukzessive global ausbreitete, zeigten die hinsichtlich der Vehemenz und Konsequenz variierenden Schutzmaßnahmen - insbesondere bezogen auf den Umgang mit schulischen Bildungsangeboten. Wenig jedoch wurden und werden die Perspektiven der Akteur*innen in Bildungsorganisationen im Sinne einer eigenaktiven Herstellung von Handlungsräumen international und kulturvergleichend in den Blick genommen. Im Zentrum des Vergleichs steht jedoch nicht die Corona-Pandemie als medizinisch-virologische Entität, sondern vielmehr die raumzeitlichen Handlungen von Kindern und Jugendlichen in Relation zu den dargebotenen Distanzierungs- und Schutzmaßnahmen - ergo die (ggf. deprivierenden oder ermöglichenden) Bildungs- und Erziehungsverhältnisse als Tertium Comparationis. Um diese Handlungen und deren (Re-)Präsentationen empirisch fassbar zu machen, unterliegen der explorativen Studie folgende drei Forschungsfragen:

1. Wie präsentieren und erzählen Kinder und Jugendliche ihre Lernsituation in Zeiten erforderlicher Distanzierung?

2. Wie stellen sie ihre Lernräume her?

3. Wie wird der Prozess der Herstellung von Lernräumen durch die vorherrschenden Bildungs- und Erziehungsverhältnisse, durch die ökonomische Entwicklung etc. in den aktuellen Zeiten erforderlicher Distanzierung bedingt?

Zur Umsetzung des Forschungsvorhabens werden Schüler*innen aus unterschiedlichen Ländern und Kulturkontexten an der Schnittstelle von formaler Bildungssituation in Schulen und informellen Lernsituationen zuhause adressiert. In jugendlicher Altersspanne zwischen 13 und 16 Jahren werden Schüler*innen u.a. aus Deutschland, Österreich, der Schweiz, Island, Georgien, Armenien und Thailand eingeladen, sich - alleine oder zu mehreren - an der ,spielerisch-digitalen Präsentation der eigenen Lernsituation zu beteiligen. Der methodische Zugang sieht vor, dass die sich beteiligenden Schüler*innen in ihren selbst gewählten Lernumgebungen fotografieren und hierzu kurzer Erläuterungen verfassen. Die Darstellung der Lernsituation kann dabei als Einzelfotografie erfolgen oder auch als szenische Abfolge mehrerer Fotografien bzw. eines kurzen Clips. Für letztere Variante wird die spielerische Methode der so genannten "Mannequin-Challenge“ vorgeschlagen, in der sich Menschen in kleineren oder größeren Gruppen so positionieren und körperlich stillhalten, so dass eine filmende Person die Szenerie als ,eingefrorenes' Stillleben aufzeichnet. Neben dem empirischen Nutzen der 
Daten und der hieraus folgenden Erkenntnisgewinnung ermöglicht das methodische Vorgehen den Jugendlichen einen potenziell spielerischen und humorvollen Umgang mit den eigenen Bedingungen und die Möglichkeit einer reflektierten Distanz via Selbstpositionierung.

Die Handlungsangebote, die an die Kinder und Jugendlichen gerichtet wurden, lauten wie folgt:

1. Mache ein Foto, mehrere Fotos oder einen kurzen Clip (Mannequin Challenge) einer derzeit typischen Lernsituation (während der Lockdown-Phase oder nach Wiederaufnahme des Schulbetriebs)!

2. Schreib ca. drei Sätze darüber, was du auf dem Foto, in der Fotoreihe oder dem Clip zeigen willst!

3. Beschreibe kurz deinen Lernort: Wo wurden die Bilder aufgenommen? Was ist zu sehen? Was hast du vor dem Fotografieren gemacht, was machst du danach?

Ziel ist somit eine Präsentation des Lernraums durch die Schüler*innen, den sie ggf. für die Foto(s) als solchen herstellen, sowie eine Beschreibung und Kontextualisierung der Situation, wodurch, unter Anwendung von rekonstruktiven Auswertungs- und Analysetechniken, implizite Raum-Praktiken der Schüler*innen und deren Relation zu materiellen und sozialen Bildungs- und Erziehungsverhältnissen und potenziell hieraus aufscheinenden Inklusionsbedingungen rekonstruiert werden können. Die erwarteten Ergebnisse beziehen sich also auf Raumherstellungen und -aneignungen von Schüler*innen vor dem Hintergrund einer abrupten und exogenen Einwirkung durch Maßnahmen zur Bekämpfung der Corona-Pandemie. Das Verhältnis von exogen bedingter Raumproduktion und schüler*innenbezogenen Raumaneignung kann dabei - ähnlich zum Habitus als wechselseitig angenommen werden (vgl. Bourdieu 2018[1991]), wodurch ein kulturbasierter Einblick in die Praktiken der Schüler*innen in unterschiedlichen Länderkontexten ermöglicht werden kann. Vor dem Hintergrund der krisenhaften Schul- und Bildungssituation können somit Erkenntnisse dahingehend abgeleitet werden, wie (inklusionsorientierte) Lernräume (weiter-) zu entwickeln sind und wie dieser Prozess auch in krisenhaften Situationen fortgeführt werden kann. Dies macht Impulse für die Diskurse um inklusions- und heterogenitätsorientierte Lernraumgestaltung sowie prekäre Lern- und Bildungsräume im Kontext vulnerabler Gruppen und sozialer Ungleichheit erwartbar. 


\section{Visuelle Perspektiven auf Körper, Raum und Inklusion/ Exklusion}

Hier wird zunächst die methodologische Perspektive visuell informierter qualitativ-rekonstruktiver (Inklusions-)Forschung innerhalb des Projekts ausgeführt und ins Verhältnis zu den theoretischen Überlegungen zu (Lern-)Raumproduktion und -körper (Kap. 2) sowie zu Inklusionsbedingungen (Kap.4) gesetzt und, nicht zuletzt, mit Blick auf die vorherrschende Corona-Pandemie erweitert.

Die im Projekt „Distant Bodies - Collective Spaces - Borders“ entstehenden visuellen Daten werden von den Schüler*innen aufgenommen. Sie bestimmen demnach die Perspektiven und möglichen Blickwinkel, aus denen die Fotografien aufgenommen und Fotogramme hergestellt werden. In der Vorbereitung auf die Präsentation eines Lernraums können sowohl die physischen Artefakte (z.B. Tische, Schreibutensilien) sowie die Körper in Position gebracht bzw. angeordnet werden. Die Schüler*innen stellen demnach nicht nur die Bilder her, sondern in Vorbereitung darauf auch die lernräumliche (Re-)Präsentation. Die hierdurch angestoßene Reflexion der Schüler*innen kann als Interaktion mit dem eigenen Lernraum angesehen werden und ermöglicht eine kreative Distanzierung zur Verfasstheit des eigenen Lernraumes. Dies wird unterstützt durch eine im Nachgang erfolgende textbasierte Selbstbeschreibung der präsentierten Lernräume und der in ihnen präsentierten Körper durch die Schüler*innen. So entstehen visuelle und textbasierte Raumbilder, d.h. mikroperspektivische und fragmentarische Konstruktionen von Lernräumen (vgl. Döring 2009).

Diese Raumbilder stellen raumzeitliche Momentaufnahmen dar (vgl. Kracauer 2017, 22). Durch die Simultanität des visuell Dargebotenen (Bohnsack 2006) und durch die kulturell wie sozial eingebundenen Produktionsverhältnisse wird Fotografien ein „Bedeutungsüberschus" (Stumberger 2010, 63) zugeschrieben, der für die Zwecke einer an Ein- und Ausschlussprozessen interessierten Sozialforschung entschlüsselt werden kann. Im Projekt „Distant Bodies - collective spaces - borders" wird durch die Selbstpositionierung der Schüler*innen zusätzlich zu einem Medium der Inszenierung gegriffen. Die Schüler*innen bilden sich in ihrer Rolle als Lernende ab und stellen ihre Lernkörper im Verhältnis zu einem durch Corona raumzeitlich transformierten Sozialraum vor. Die in der Inszenierung performierte Rolle - und hier schließt Goffmans (2001) Sozial- bzw. Rollentheorie an - kann die an die Schüler*innen gerichteten Erwartungen und Anforderungen an Lernen in Zeiten erforderlicher Distanz vor dem Hintergrund zur Verfügung stehender Ressourcen und Bedingungen sichtbar machen. Ergo ist es das Ziel, die Fotografien und Fotogramme in erster Linie als Momentaufnahmen zu behandeln, die die Lernenden zur Präsentation ihrer Lernsituation selbst ausgewählt haben. Es interessiert also, welchen Einblick die Lernenden zeigen, was sie nach außen tragen im Sinne eines, Abbildes` einer sozialen und habituellen Praxis. Das 
Dargestellte kann daher hinsichtlich seiner Konstruktion, Entstehung und späteren Distribution dechiffriert werden. Durch die Simultanität des Abgebildeten bietet die Fotografie ein hohes Maß an Geschlossenheit und rezeptionslogischer Deckung mit einer situationalen Erfahrung, da die Fotografien und Fotogramme „ein präzises Abbild [...] liefern, ohne das Vorbild imaginativ konsumieren zu müssen" (ebd.). Diese Analyseperspektive, mit der die Intention verbunden ist, aus einer fotografisch-bildlichen Repräsentation von Welt und Wirklichkeit auf die strukturelle Grammatik des Ganzen zu schließen, wurde in den vergangenen Jahren insbesondere in der visuellen Soziologie eingenommen (Breckner \& Raab 2016). In methodologischer Hinsicht ist diesen visuellen Zugängen also das Potential inhärent, die „Praxis des Sehens“ (Prinz 2014) zu rekonstruieren und hierdurch einen reflexiven Blick auf die Darstellungen des Raumes zu erhalten und zu entschlüsseln, welche Raumhandlungen im Bild enthalten sind (vgl. Kracauer 2017[1963], 36; Panofsky 2006).

\section{Perspektiven}

In diesem Beitrag wurde entlang raum- und inklusionstheoretischer Überlegungen ein Blick auf die durch die Corona-Pandemie hervorgerufene veränderte Lernsituation von Schüler*innen gerichtet. Es wurde deutlich gemacht, dass die ,Verräumlichung' formaler Bildung im Sinne einer Suspendierung routinehafter Bildungsprozesse in Bildungsorganisationen als globales Phänomen anzusehen ist, das Schüler*innen vor Herausforderungen stellt - und ebenso die erziehungswissensschaftliche Forschung, diese ,neuen' Erfahrungen empirisch zu fassen.

Dabei wurde eine performative Perspektive auf Raum deutlich, in der sozialer wie physischer Raum zwar als präformiert im Sinne einer lenkenden aber nicht determinierenden Entität erachtet wird, der proaktiv von den Schüler*innen angeeignet wird. So wird der veränderte Lernraum durch die Schüler*innen mit hergestellt. Dies bietet empirischen Zugängen die Möglichkeit, die Herstellung und Aneignung von Raum - und die darin enthaltenen Handlungsräume/Agency sowie potenzielle Inklusionsbedingungen - sichtbar zu machen. In den Interaktionen der Schüler*innen mit dem eigenen Lernraum, die sich in der visuell dokumentierten Selbst-Positionierung ausdrücken, werden demnach Reaktionen auf die Suspendierung perspektiviert. Vor diesem Hintergrund werden durch die

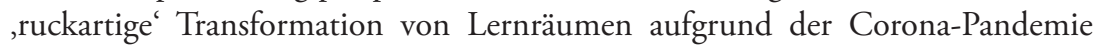
Inklusionsbedingungen für Schüler*innen neu sichtbar - in veränderten Raumkonstellationen und vor dem Hintergrund sozio-kultureller Bedingungen. Im Projekt „Distant Bodies - Collective Spaces - Borders“ sind dafür visuelle und performative Zugänge zentral, in denen Schüler*innen die Positionierung und 
Visualisierung ihrer Lernraumsituation ermöglicht wird. So werden - kulturraumvergleichend - mögliche Dimensionen des lernraumbezogenen Handelns sichtbar gemacht, z.B. zwischen Privatheit und Öffentlichkeit, Selbstorganisation und (technischer) Funktionalisierung, Digitalem und Analogem (vgl. Köpfer, Lemmer \& Rißler 2021).

\section{Literatur}

Alkemeyer, Thomas (2006): Lernen und seine Körper. Habitusformungen und -umformungen in Bildungspraktiken. In: Friebertshäuser, Barbara, Riegger, Markus \& Wigger, Lothar (Hrsg.): Reflexive Erziehungswissenschaft. Wiesbaden, 119-141.

Andresen, Sabine, Lips, Anna, Möller, Renate, Rusack, Tanja, Schröer, Wolfgang, Thomas, Severine \& Wilmes, Johanna (Hrsg.) (2020): Erfahrungen und Perspektiven junger Menschen während der Corona-Maßnahmen. Hildesheim.

Bohnsack, Ralf (2006): Die dokumentarische Methode der Bildinterpretation in der Forschungspraxis. In: Marotzki, Winfried \& Niesyto, Horst (Hrsg.): Bildinterpretation und Bildverstehen. Methodische Ansätze aus sozialwissenschaftlicher, kunst- und medienpädagogischer Perspektive, Wiesbaden, 45-75.

Ahrens, Daniela (2009): Der schulische Lernort: Zwischen institutioneller Entgrenzung und sozialer Verräumlichung. In: Böhme, Jeanette (Hrsg.): Schularchitektur im interdisziplinären Diskurs. Wiesbaden, 73-87.

Allan, Julie (2004): Deterritorializations: Putting postmodernism to work on teacher education and inclusion. In: Educational Philosophy and Theory, 36, H.4, 417-432.

Bourdieu, Pierre (2016 [1985]): Sozialer Raum und Klassens: Lecon sur la lecon. Zwei Vorlesungen. Frankfurt am Main.

Bourdieu, Pierre (2018 [1991]): Social Space and the Genesis of Appropriated Physical Space. In: International Journal of Urban and Regional Research, 42, H.1, 106-114.

Breckner, Roswitha \& Raab, Jürgen (2016): Materiale Visuelle Soziologie. In: Zeitschrift für Qualitative Forschung, 17, H.1-2, 5-9.

Bremm, Nina \& Rachenbäumer, Kathrin (2020): Dimensionen der (Re-)Produktion von Bildungsbenachteiligung in sozialräumlich deprivierten Schulen im Kontext der Corona-Pandemie. In: Die Deutsche Schule, 16. Beiheft, 202-215.

Bruner, Claudia F. (2005): Körper und Behinderung im Diskurs. Empirisch fundierte Anmerkungen zu einem kulturwissenschaftlichen Verständnis der Disability Studies. In: Psychologie und Gesellschaftskritik, 29, H.1, 33-53.

Döring, Jörg (2009): Raumbilder 1930. Siegfried Kracauers spatiale Hermeneutik für die Frankfurter Zeitung. In: Schröter, Jens, Winter, Gundolf \& Barck, Johanna (Hrsg.): Das Raumbild. Bilder jenseits ihrer Flächen. München, 331-350.

Döring, Jörg \& Thielmann, Tristan (Hrsg.) (2008): Spatial Turn. Das Raumparadigma in den Kulturund Sozialwissenschaften. Bielefeld.

Glaser, Edith, Koller, Hans-Christoph, Thole, Werner \& Krumme, Salome (Hrsg.) (2018): Räume für Bildung - Räume der Bildung. Opladen, Berlin, Toronto.

Goffman, Erving (2001): Wir alle spielen Theater. München.

Gogolin, Ingrid (2020): Sprachliche Förderung, sprachliche Bildung und Lernen im Deutschen als Zweitsprache während und nach der Pandemie. In: Die Deutsche Schule, 16. Beiheft, 175-188.

Goldan, Janka, Geist, Sabine \& Lütje-Klose, Birgit (2020): Schüler*innen mit sonderpädagogischem Förderbedarf während der Corona-Pandemie. In: Die Deutsche Schule, 16. Beiheft, 189-201. 
Hirschberg, Marianne \& Koebsell, Swantje (2021): Disability Studies in Education: Normalität/en im inklusiven Unterricht und im Bildungsbereich hinterfragen. In: Köpfer, Andreas, Powell, Justin J.W. \& Zahnd, Raphael (Hrsg.): Handbuch Inklusion international. Opladen, Berlin, Toronto, 127-146.

Hummrich, Merle (2012): Jugend und Raum. Exklusive Zugehörigkeitsordnungen in Familie und Schule. Wiesbaden.

Hummrich, Merle (2009): Exklusive Zugehörigkeit. Eine raumanalytische Betrachtung von Inklusion und Exklusion in der Schule. In: sozialersinn, 10, H.2, 3-32.

Köpfer, Andreas, Papke, Katharina \& Gerdes, Jürgen (2019): Rekonstruktionen zum Verhältnis von Inklusionsverständnissen und -bedingungen praktizierender Lehrkräfte. In: Journal für Psychologie, 27, H.2, 170-191.

Köpfer, Andreas, Krause, Sabine, Proyer, Michelle \& Zahnd, Raphael: Forschungsprojekt „Distant Bodies - Collective Spaces - Borders“. Online. www.distantbodies.eu (Stand: 12.02.2021).

Köpfer, Andreas, Lemmer, Kathrin \& Rißler, Georg (2021): Raumrelationen von Inklusion und Exklusion im Handeln der Schüler*innen - Ein mehrdimensionaler Zugang zum Verhältnis von materialem und sozialem Raum in Bildungsorganisationen. In: Tertium Comparationis, H.3, [im Erscheinen].

Kracauer, Siegfried (2017 [1963]): Die Photographie. In: ders. (Hrsg.): Das Ornament der Masse. Frankfurt am Main, 21-39.

Löw, Martina (2001): Raumsoziologie. Frankfurt am Main.

Nentwig-Gesemann, Ines (2007): Sprach- und Körperdiskurse von Kindern. In: Friebertshäuser, Barbara, Felde, Heike von \& Schaffer, Burkhard (Hrsg.): Bild und Text. Opladen, 105-120.

Panofsky, Erwin (2006): Ikonographie und Ikonologie: Bildinterpretation nach dem Dreistufenmodell. Köln.

Prinz, Sophia (2014): Die Praxis des Sehens. Bielefeld.

Schatzki, Theodor R. (2019): Social Change in a Material World. Milton Park.

Schroer, Markus (2012): Räume, Orte, Grenzen. Frankfurt am Main.

Schroer, Markus (2003): Körper und Raum - Grenzverläufe. In: Leviathan, 31, 401-416.

Sliwka, Anne \& Klopsch, Britta (2020): Disruptive Innovation! In: Die Deutsche Schule, 16. Beiheft, 216-229.

Soja, Edward W. (2008): Vom „Zeitgeist“ zum „Raumgeist“. In: Döring, Jörg \& Thielmann, Tristan (Hrsg.): Spatial Turn. Das Raumparadigma in den Kultur- und Sozialwissenschaften. Bielefeld, 241-262.

Soja, Edward W. (1985): The Spatiality of Social Life: Towards a Transformative Retheorisation. In: Gregory, Derek \& Urry, John (Hrsg.): Social Relations and Spatial Structures. Macmillan: Houndsmills, 90-127.

Stumberger, Rudolf (2010): Das Bild unter der Schneedecke. Visuelle Soziologie: Erforschung des Sozialen mit anderen Mitteln. In: Forschung Frankfurt, 26, H.2, 62-66.

Weisser, Jan (2005): Theorie der Behinderung. Bielefeld.

Weisser, Jan (2017): Konfliktfelder schulischer Inklusion/Exklusion im 20. Jahrhundert. Weinheim.

Wulf, Christoph \& Zirfas, Jörg (2014): Handbuch Pädagogische Anthropologie. Wiesbaden. 